\title{
Metastatic Follicular Thyroid Carcinoma to the Dorsal Spine Presenting as Early Manifestation: Case Report
}

\author{
Romit Agrawal*, Ayush Sharma, Vijay Singh, Nilesh Mangale and \\ Ajay Jaiswal \\ Department of Orthopaedics and Spine Surgery, Dr B.R Ambedkar Central Railway \\ Hospital, Byculla, Mumbai, India \\ *Corresponding Author: Romit Agrawal, Department of Orthopaedics and Spine \\ Surgery, Dr B.R Ambedkar Central Railway Hospital, Byculla, Mumbai, India.
}

\author{
Received: March 25, 2020 \\ Published: April 10, 2020 \\ (C) All rights are reserved by Romit
}

Agrawal., et al.

\begin{abstract}
Follicular thyroid carcinoma (FTC) patients are rarely present with spinal metastasis as an early manifestation. Here we present a 56 year old non ambulatory female with paraparesis of both lower limbs which was progressed over last 3 months. Spinal MRI and CT scan revealed D7 thoracic spine intramedullary lesion probably of thyroid origin which was confirmed on USG and FNAC later on. The aim of surgery was decompression of spinal cord and histological evaluation of the tumour. Surgery in the form of spinal mass excision and posterior spinal decompression and fixation two levels above and below was done and then adjuvant chemotherapy was given. Hence patients presenting with spinal tumour must always be screened for thyroid carcinoma as a differential diagnosis. Prognosis is quite well in such patients post decompression.

Keywords: Follicular Type Thyroid Carcinoma; Spinal Metastasis; Spinal Tumour
\end{abstract}

\section{Abbreviations}

FTC: Follicular Thyroid Carcinoma; MRI: Magnetic Resonance Imaging; CT: Computed Tomography; USG: Ultrasonography; FNAC: Fine Needle Aspiration Cytology; PET: Positron Emission Tomography; DTC: Differentiated Thyroid Carcinoma; TIRADS: Thyroid Imaging Reporting and Data System; TMC RSS: Thyroid Multimodal-Imaging Comprehensive Risk Stratification Scoring; TTF-1: Thyroid Transcription Factor 1; RAI: Radioactive Iodine

\section{Introduction}

Follicular thyroid carcinoma is the second most common type of Differentiated thyroid carcinoma [1]. It commonly spreads to bone due to its hematogenous spread [2]. FTC involves the bones in $7-12 \%$ of the cases [3]. These metastases can accumulate in the osseous elements in the spine and expand to epidural space by variable degrees. Thoracic spine and lumbar spine are the most preferable sites for the metastatic tumours [4]. The neoplastic mass progressively substitutes the bone tissue thereby destructing the structural elements in spine which results into loss of stability and compression of the intra-canal neural structures [5]. Spinal cord compression due to metastatic follicular thyroid cancer mainly occurs in the later stage of the disease [6]. However, cord compression as the initial presentation of FTC is very rare [7]. We present such rare case that was admitted with thoracic spinal metastasis and first presented with symptoms of cord compression without any symptom related to the primary thyroid cancer and later on follicular thyroid cancer was diagnosed after pathological evaluation of the spinal tumour. We stress on the need for appropriate evaluation of thyroid for a diagnosed case of spinal metastases of unknown origin.

\section{Case Report}

A 56 Year old female with history of hypertension presented to our outpatient clinic with 3 months old history of back pain radiating to both lower limbs along with tingling and numbness. She had developed progressive weakness in both lower limbs more on the left side without urinary incontinence. Pain was exacerbated by sitting and improved on lying down position. On physical examination, we noted significant bilateral clonus and strength of $1+/ 5$ in her right and 1/5 in left lower extremities. On thoracic computerized tomography (CT) examination, an osteolytic lesion with moderate lobulated soft tissue component $2.7^{*} 3.7 \mathrm{~cm}$ in axial plane and $3.4 \mathrm{~cm}$ in superioinferior extent noted at D6-D7 vertebral level causing significant destruction of the D7 pedicle on the left side, bilateral lamina and spinous process (Figure 1). Large soft tissue density mass lesion with areas of calcification was seen on the left side of neck suggestive of thyroid lesion. Magnetic resonance imaging (MRI) of spine showed strongly enhancing lesion in posterior elements of D7 vertebra. The lesion had encroached the spinal canal, causing displacement and cord compression (Figure 2). The lesion was hypermetabolic at D6 and D7 vertebra on positron emission tomography (PET) CT and there were also a hypermetabolic enhancement in the left thyroid lobe. Neck palpation revealed a firm solitary nodule in left lobe of the thyroid. USG evaluation of the neck showed left thyroid nodule with TIRADS scores 4C and TMC RSS score 5: high risk was noted. Laboratory results including complete blood cell counts, erythrocyte sedimentation rate, C-reactive protein, etc. were within normal ranges. Due to acute neurological worsening, the patient was given intravenous (IV) corticosteroids to reduce edema of the spinal cord. Moreover, the therapeutic goal for this patient was spinal cord decompression and stabilization. 
Unfortunately, the patient declined the option of undergoing percutaneous biopsy of the vertebral lesion but consented to surgical resection. As these was the only metastatic lesion apparent at the time of work up on PET scan, so complete resection of the lesion with decompression of cord was planned. Pre-operatively embolization of spinal metastasis was done and surgical decompression was planned within 24 hours of embolization. Standard posterior approach was used, placement of pedicle screws from D5 through D9, excluding D7 was done (Figure 3 and 5). Laminectomies of D6 and D7 revealed a highly vascular mass displacing the spinal cord posteriorly and to the left. Intralesional excision of tumour along with debulking with decompression and spinal fixation was done (Figure 4). There was no residual tumour on postoperative MRI (Figure 6). Histological examination revealed metastasis of differentiated thyroid carcinoma displaying follicular pattern.

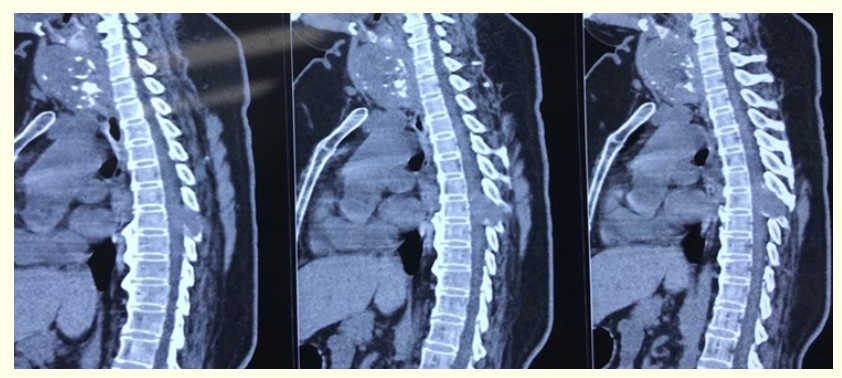

Figure 1: Sagittal sections of the dorsal spine computerized

tomography demonstrating lytic posterior elements at D7 vertebral level causing destruction of left pedicle and both lamina.

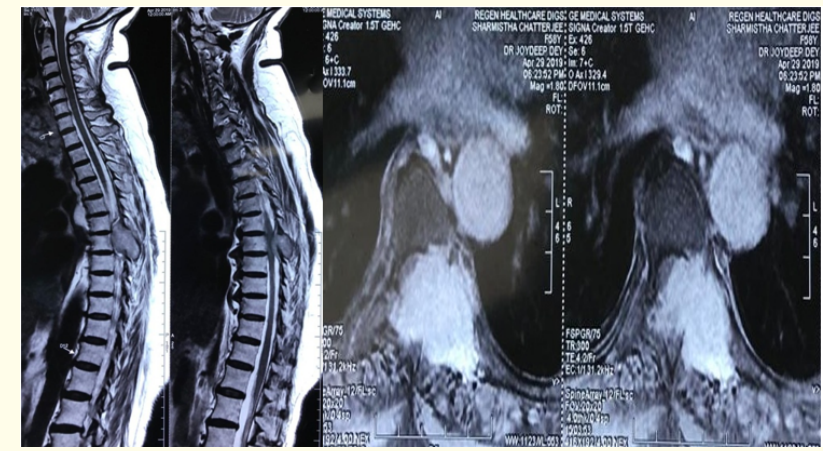

Figure 2: Sagittal and axial sections of the T2-wieghted dorsal spinal magnetic resonance imaging demonstrating heterogeneous hypo- and hyperintense tumor in the posterior elements at D7 vertebra level compressing the spinal cord.

Immunohistochemical investigations revealed that the tumour cells were positive for PAX-8 and thyroid transcription factor 1 (TTF-1). These findings verified the diagnosis of DTC. Later on USG guided FNAC was performed which showed many clusters of thyroid follicular cells with nuclear overlapping, enlargement and occasional nuclear grooves with atypical follicular cells, suggestive of follicular neoplasm. Bethesda category 4 Patient was then transferred to the ENT department and underwent total thyroidectomy along with I 131 radioablation after 6 weeks post-surgery. Histo-

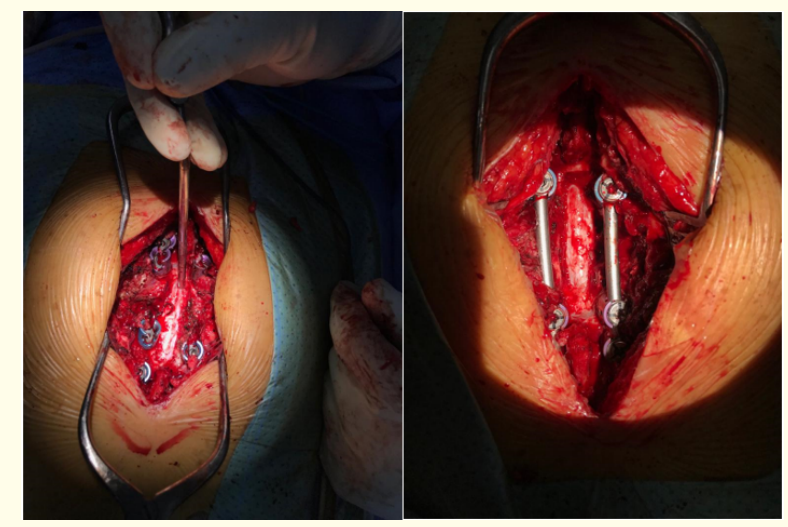

Figure 3: Clinical picture showing laminectomy and well decompressed spinal cord with stabilization of spine.

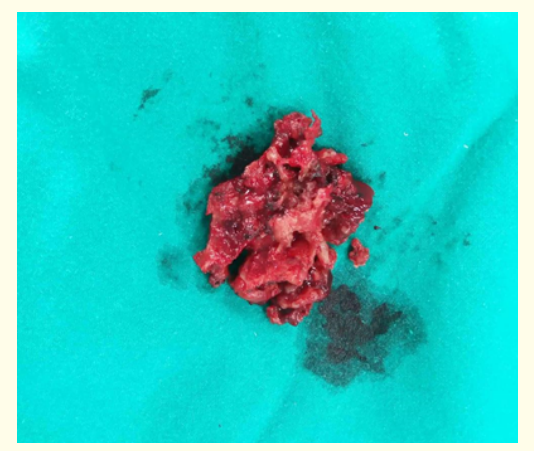

Figure 4: Clinical picture showing excised mass which was compressing the spinal cord.

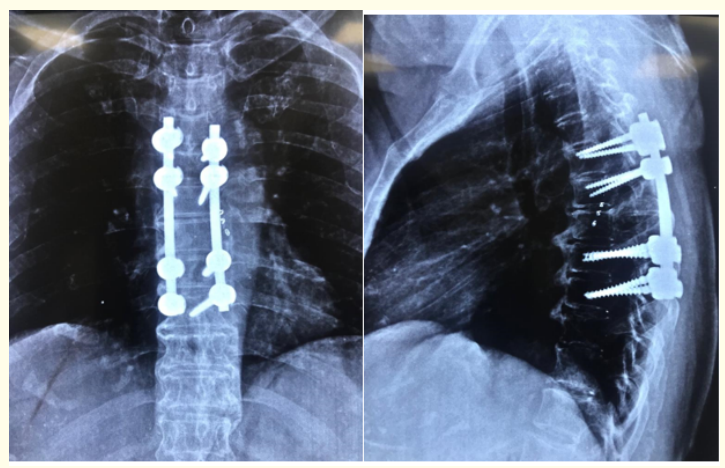

Figure 5: Post-operative radiograph showing stabilization of spine from D5-D9 excluding D7.

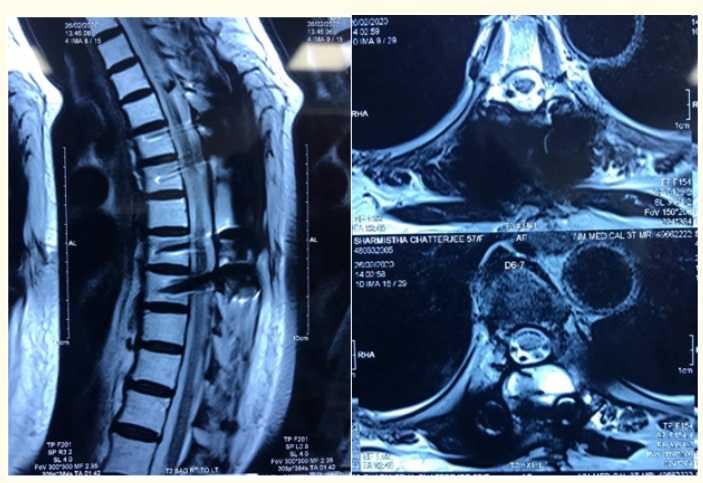

Figure 6: Post-operative MRI sagittal and axial sections showing well decompressed spinal cord. 
pathological examination of the surgical specimen confirmed the follicular thyroid carcinoma. Post-operative period was uneventful. She has also received few cycles of chemotherapy for the same and on follow up of 6 months, strength was improved to $5 / 5$ in the bilateral lower extremities, and she reported a reduction in back pain. There was no recurrence or new metastasis on 1 year followup.

\section{Discussion}

Among differentiated thyroid carcinoma, follicular thyroid carcinoma spreads through hematogenous route to bone, lung and central nervous system [8]. Incidence of bony metastasis of FTC was reported as 7 to $12 \%$ and of spinal metastasis as 1 to $7 \%$ [9]. It is observed that follicular thyroid carcinomas have already been metastasized distantly before causing any symptoms from primary thyroid lesion [10]. Symptoms of Spinal cord compression as initial presentation are very uncommon event. Review of literature shows only few cases of spinal cord compression presenting as the initial symptoms of follicular thyroid carcinoma. Pomorski and Bartos [11] in there study of 309 cases of FTC; they reported only one case of distant spinal metastasis as an initial presentation. 10 year survival rate of the DTCs is as high as $80-95 \%$. However, in patients with bony distant metastases, survival rate is observed to be reduced [12]. It is also observed that long-term survival with distant metastasis as an initial presentation is quite high as $44 \%$. But presence of spinal metastases reduces quality of life due to severe pain and/or neurological deficits $[13,14]$. In patients presenting with a spinal mass of unknown origin, it may be a diagnostic challenge to raise a suspicion of an occult thyroid cancer. Therefore, careful history taking and thorough physical examination becomes mandatory. In our case CT scan of dorsal spine with whole spine screening was done as an initial investigation which incidentally picked up the synchronous lesion in the thyroid gland along with destruction of posterior elements at D7 vertebra level. USG neck was done to confirm the CT findings which showed left thyroid nodule with TIRADS scores 4C and TMC RSS score 5 which confirm the primary thyroid malignancy. Later on MRI confirmed the diagnosis with an intramedullary extradural mass compressing the spinal cord at D7 level. Early recognition of the primary source of metastatic spinal disease is very important as functional outcome after resection of mass compressing the spinal cord depends on neurologic condition at the time of presentation. After confirming the source of distant spinal metastasis, it becomes mandatory to intervene therapeutically as soon as possible to alleviate pain, preserve or improve neurologic function, achieve mechanical stability, optimize primary and metastatic site tumour control and hence improved quality of life. Literature doesn't show any protocol for the management of the spinal metastases in well differentiated thyroid cancer as only small case series are available. Spinal metastases of the DTCs are resistant to radiotherapy and moreover they are usually isolated metastases hence total resection is recommended [14]. Spinal metastasis embolization of the of DTCs 24 hours before operation may be a wise option because of tendency of these tumors to profusely bleed intraopera- tively [15]. Sellin., et al. [16] in his study of factors affecting survival in 43 consecutive patients after surgery for spinal metastases from thyroid carcinoma found that preoperative embolization was significantly associated with fewer complications. Spinal metastasis causing compression of cord should be treated preferentially with enblock resection of mass along with decompression of spinal cord and stabilization. In case of recurrence of metastasis, Stojadinovic., et al. [17] suggested a surgery as the preferred method of treatment for resectable recurrence, followed by radioactive iodine (RAI) therapy for iodide-concentrating thyroid cancer, or externalbeam radiation for tumors that lack RAI avidity. Similarly, Proye., et al. showed that differentiated thyroid cancer is usually less life threatening and that early diagnosis and appropriate treatment for distant metastases can significantly prolong the life span and improve the quality of life [18].

\section{Conclusion}

In conclusion, Follicular thyroid carcinoma presenting with spinal metastasis as initial finding is very uncommon therefore thyroid screening must be kept in mind in the cases of spinal metastasis in which primary tumour site could not be found. Patients with FTC with spinal metastases has reduced long term survival rate compared to patients without spinal metastasis. Surgical decompression of spinal metastases in patients with significant cord compression along with total/near total thyroidectomy and radioactive iodine treatment may decrease the recurrence rate and provides good quality of life.

\section{Conflict of Interest}

No financial interest or conflict of interest exists.

\section{Bibliography}

1. Carhill AA., et al. "Durable effect of radioactive iodine in a patient with metastatic follicular thyroid carcinoma". Endocrinology (2012): 231912.

2. McNeely MF., et al. "Follicular thyroid carcinoma presenting as acute cord compression due to thoracic vertebral metastasis". Radiology Case Reports 7 (2012): 1-4.

3. Pacini F., et al. "European consensus for the management of patients with differentiated thyroid carcinoma of the follicular epithelium". European Journal of Endocrinology 154.6 (2006): 787-803.

4. Laufer I., et al. "Surgical management of metastatic spinal tumors". Cancer Control 19.2 (2012): 122-128.

5. Gasbarrini A., et al. "Spinal metastases: treatment evaluation algorithm". European Review for Medical and Pharmacological Sciences 8.6 (2004): 265-274.

6. Hsiao FC., et al. "Metastatic spinal cord compression as initial presentation of occult follicular thyroid carcinoma". Journal of Medical Sciences 28 (2008): 89-94. 
7. Kim DK., et al. "Spinal cord compression as initial presentation of follicular thyroid carcinoma". Journal of Korean Neurosurgical Society 41.4 (2007): 269-271.

8. Robbins J., et al. "Thyroid cancer: a lethal endocrine neoplasm". Annals of Internal Medicine 115.2 (1991): 133-147.

9. Marcocci C., et al. "Clinical and biologic behavior of bone metastases from differentiated thyroid carcinoma”. Surgery 106.6 (1989): 960-966.

10. Burrow GN. "The thyroid nodules and neoplasia". Endocrinology and Metabolism. New York: McGraw-Hill (1995) 521-553.

11. Pomorski L and Bartos M. "Metastasis as the first sign of thyroid cancer". Neoplasma 46.5 (1999): 309-312.

12. Kim DH., et al. "Thyroid cancer initially presenting compression fracture without common thyroid symptoms". Annals of Rehabilitation Medicine 36.5 (2012): 735-738.

13. Shaha AR., et al. "Differentiated thyroid cancer presenting initially with distant metastasis". American Journal of Surgery 174.5 (1997): 474-476.

14. Kushchayeva YS., et al. "Current treatment modalities for spinal metastases secondary to thyroid carcinoma". Thyroid 24.10 (2014): 1443-1455.

15. Chander A., et al. "Surgical management of follicular carcinoma of thyroid with spinal metastasis". International Surgery Journal 2.4 (2015): 599-603.

16. Sellin JN., et al. "Factors affecting survival in 43 consecutive patients after surgery for spinal metastases from thyroid carcinoma”. Journal of Neurosurgery: Spine 23.4 (2015): 419-428.

17. Stojadinovic A., et al. "The role of operations for distantly metastatic well-differentiated thyroid carcinoma". Surgery 131.6 (2002): 636-643.

18. Proye CA., et al. "Is it still worthwhile to treat bone metastases from differentiated thyroid carcinoma with radioactive iodine?" World Journal of Surgery 16.4 (1992): 640-645.

\section{Assets from publication with us}

- Prompt Acknowledgement after receiving the article

- Thorough Double blinded peer review

- Rapid Publication

- Issue of Publication Certificate

- High visibility of your Published work

Website: https://www.actascientific.com/

Submit Article: https://www.actascientific.com/submission.php Email us: editor@actascientific.com

Contact us: +919182824667 